\title{
Letter to the editor on: "Second-trimester and third-trimester maternal lipid profiles significantly correlated to LGA and macrosomia"
}

\author{
Rui-Hong Xue ${ }^{1}$ (D) \\ Received: 7 March 2021 / Accepted: 27 April 2021 / Published online: 8 May 2021 \\ (c) The Author(s), under exclusive licence to Springer-Verlag GmbH Germany, part of Springer Nature 2021
}

\section{Dear Editor,}

We read with great interest the article by Xi et al. entitled "Second-trimester and third-trimester maternal lipid profiles significantly correlated to LGA and macrosomia" [1]. The retrospective study concluded that high TG levels during the second or third trimester could be both considered as indicators of a high risk of large for gestational age (LGA) and macrosomia, and suggested that future lifestyle programs on lowering TG levels may help to reduce the incidence of LGA and macrosomia. However, the authors did not consider high TG effects during the first trimester, which might have partly contributed these increased risks of LGA and macrosomia. We would like to address some points that merit more attention.

Elevated triglyceride (TG) levels during early or late pregnancy have been reported both associated with increased risks of adverse pregnancy outcomes. For years, clinical interventions during gestation to reduce adverse pregnancy have been reported not very ideal, as was reported [2], the lifestyle intervention in pregnancy had no measurable effect on obstetrical or neonatal outcomes, despite a modest but significant decrease in gestational weight gain (GWG).

In our recent published research, we found that high TG levels during early pregnancy was associated with increased risks of preterm delivery, preeclampsia, GDM and LGA, However, compared with high TG levels during early pregnancy alone, persistently high TG levels during both early and late pregnancy only slightly increased about $30 \%$ risks of LGA without increasing risks of preeclampsia and GDM,

This comment refers to the article available online at https://doi. org/10.1007/s00404-021-06010-0.

Rui-Hong Xue

wfmcxrh@sjtu.edu.cn

1 Department of Obstetrics and Gynecology, International Peace Maternity and Child Health Hospital, School of Medicine, Shanghai Jiao Tong University, No. 910 Hengshan Road, Shanghai 200030, China which indicated that elevated TG levels during early not late pregnancy could be the crucial factor associated with adverse pregnancy outcomes [3]. Women with pre-pregnancy overweight (OW) and obesity (OB) presented higher means of TG levels during gestation, compared to normal weight (NW) women [4]. We think OW and OB women with prepregnancy lifestyle interventions to prevent the appearance of high TG levels during early pregnancy may benefit more in reducing the risks of adverse pregnancy outcomes, rather than only focus on interventions during gestation.

Author contributions RHX conceived and wrote the manuscript.

Funding None.

\section{Declarations}

Conflict of interest The authors report no conflicts of interest.

\section{References}

1. Xi F, Chen H, Chen Q et al (2021) Second-trimester and thirdtrimester maternal lipid profiles significantly correlated to LGA and macrosomia. Arch Gynecol Obstet. https://doi.org/10.1007/ s00404-021-06010-0

2. Sagedal LR, Øverby NC, Bere E et al (2017) Lifestyle intervention to limit gestational weight gain: the Norwegian Fit for Delivery randomised controlled trial. Bjog Int J Obstetr Gynaecol 124:97-109

3. Xue RH, Wu DD, Zhou CL et al (2021) Association of high maternal triglyceride levels early and late in pregnancy with adverse outcomes: a retrospective cohort study. J Clin Lipidol 15(1):162-172

4. Farias DR, Franco-Sena AB, Vilela A et al (2016) Lipid changes throughout pregnancy according to pre-pregnancy BMI: results from a prospective cohort. BJOG 123:570-578. https://doi.org/ 10.1111/1471-0528.13293

Publisher's Note Springer Nature remains neutral with regard to jurisdictional claims in published maps and institutional affiliations. 